\title{
Least energy solutions for a quasilinear Schrödinger equation with potential well
}

Yujuan Jiao*

"Correspondence:
jsjyj@xbmu.edu.cn
College of Mathematics and
Computer Science, Northwest
University for Nationalities, Lanzhou,
730124, P.R. China
College of Mathematics and
Statistics, Northwest Normal
University, Lanzhou, 730070,
P.R. China

${ }^{*}$ Correspondence:

jsjyj@xbmu.edu.c 730124, P.R. China

College of Mathematics and

P.R. China

\begin{abstract}
In this paper, we consider the existence of least energy solutions for the following quasilinear Schrödinger equation:

$$
-\Delta u+(\lambda a(x)+1) u-\frac{1}{2}\left(\Delta|u|^{2}\right) u=f(u), \quad x \in \mathbb{R}^{N},
$$

with $a(x) \geq 0$ having a potential well, where $N \geq 3$ and $\lambda>0$ is a parameter. Under suitable hypotheses, we obtain the existence of a least energy solution $u_{\lambda}$ of $\left(E_{\lambda}\right)$ which localizes near the potential well int $a^{-1}(0)$ for $\lambda$ large enough by using the variational method and the concentration compactness method in an Orlicz space. MSC: 35J60; 35B33

Keywords: quasilinear Schrödinger equation; least energy solution; Orlicz space; concentration compactness method; variational method

\section{Introduction}

Let us consider the following quasilinear Schrödinger equation:

$$
-\triangle u+(\lambda a(x)+1) u-\frac{1}{2}\left(\Delta|u|^{2}\right) u=f(u), \quad x \in \mathbb{R}^{N},
$$

for sufficiently large $\lambda$, where $N \geq 3$.

Our assumptions on $a(x)$ are as follows:

$\left(a_{1}\right) a(x) \in C\left(\mathbb{R}^{N},[0,+\infty)\right)$, the potential well $\Omega:=\operatorname{int} a^{-1}(0)$ is a non-empty set and $\bar{\Omega}=$ $a^{-1}(0)$

$\left(a_{2}\right)$ There exists a constant $M_{0}>0$ such that $\mu\left(\left\{x \in \mathbb{R}^{N} \mid a(x) \leq M_{0}\right\}\right)<\infty$, where $\mu$ denotes the Lebesgue measure on $\mathbb{R}^{N}$.

Condition $\left(a_{2}\right)$ is very weak in dealing with the operator $-\Delta+(\lambda a(x)+1) I$ on $\mathbb{R}^{N}$, which was firstly used by Bartsch and Wang [1] in dealing with the semilinear Schrödinger equation.

Remark 1.1 $\Omega:=\operatorname{int} a^{-1}(0)$ can be unbounded.

For $f(u)$, we assume that $f$ is continuous and satisfies the following conditions:

$\left(f_{1}\right) \lim _{u \rightarrow 0^{+}} \frac{f(u)}{u}=0$;

(C) 2013 Jiao; licensee Springer. This is an Open Access article distributed under the terms of the Creative Commons Attribution License (http://creativecommons.org/licenses/by/2.0), which permits unrestricted use, distribution, and reproduction in any medium, provided the original work is properly cited. 
$\left(f_{2}\right) \quad 0 \leq f(u) \leq C\left(1+u^{p}\right)$ for $u \geq 0$, where $C>0$ is a constant and $4<p+1<2 \cdot 2^{*}$, where $2^{*}=\frac{2 N}{N-2}$

$\left(f_{3}\right)$ There is a number $4<\theta \leq p+1$ such that for all $u>0$, we have $f(u) \cdot u \geq \theta F(u)$, where $F(u)=\int_{0}^{u} f(t) d t$.

Hypotheses $\left(a_{1}\right),\left(a_{2}\right)$ and $\left(f_{1}\right),\left(f_{2}\right),\left(f_{3}\right)$ will be maintained throughout this paper.

Solutions of $\left(E_{\lambda}\right)$ are related to the existence of the standing wave solutions of the following quasilinear Schrödinger equation:

$$
i \partial_{t} z=-\Delta z+V(x) z-f\left(|z|^{2}\right) z-k \Delta h\left(|z|^{2}\right) h^{\prime}\left(|z|^{2}\right) z, \quad x \in \mathbb{R}^{N}
$$

where $V(x)$ is a given potential, $k$ is a real constant and $f, h$ are real functions. We would like to mention that (1.1) appears more naturally in mathematical physics and has been derived as models of several physical phenomena corresponding to various types of $h$. For instance, the case $h(s)=s$ was used for the superfluid film equation in plasma physics by Kurihara [2] (see also [3]); in the case of $h(s)=(1+s)^{\frac{1}{2}},(1.1)$ was used as a model of the self-changing of a high-power ultrashort laser in matter (see [4-7] and references therein).

In recent years, much attention has been devoted to the quasilinear Schrödinger equation of the following form:

$$
-\triangle u+\lambda V(x) u-k \triangle\left(u^{2}\right) u=|u|^{p-2} u, \quad x \in \mathbb{R}^{N} .
$$

For example, by using a constrained minimization argument, the existence of positive ground state solution was proved by Poppenberg, Schmitt and Wang [8]. Using a change of variables, Liu, Wang and Wang [9] used an Orlicz space to prove the existence of soliton solution of (1.2) via the mountain pass theorem. Colin and Jeanjean [10] also made use of a change of variables but worked in the Sobolev space $H^{1}\left(\mathbb{R}^{N}\right)$, they proved the existence of a positive solution for (1.2) from the classical results given by Berestycki and Lions [11]. By using the Nehari manifold method and the concentration compactness principle (see [12]) in the Orlicz space, Guo and Tang [13] considered the following equation:

$$
-\triangle u+(\lambda a(x)+1) u-\frac{1}{2}\left(\Delta|u|^{2}\right) u=u^{p}, \quad u>0, x \in \mathbb{R}^{N},
$$

with $a(x) \geq 0$ having a potential well and $4<p+1<2 \cdot 2^{*}$, where $2^{*}=\frac{2 N}{N-2}$ is the critical Sobolev exponent, and they proved the existence of a ground state solution of (1.3) which localizes near the potential well int $a^{-1}(0)$ for $\lambda$ large enough. In [14], Guo and Tang also considered ground state solutions of the corresponding quasilinear Schrödinger systems for (1.3) by the same methods and obtained similar results. For the stability and instability results for the special case of (1.2), one can also see the paper by Colin, Jeanjean and Squassina [15].

It is worth pointing out that the existence of one-bump or multi-bump bound state solutions for the related semilinear Schrödinger equation (1.3) for $k=0$ has been extensively studied. One can see Bartsch and Wang [1], Ambrosetti, Badiale and Cingolani [16], Ambrosetti, Malchiodi and Secchi [17], Byeon and Wang [18], Cingolani and Lazzo [19], Cingolani and Nolasco [20], Del Pino and Felmer [21, 22], Floer and Weinstein [23], Oh [24, $25]$ and the references therein. 
In this paper, based on the idea from Liu, Wang and Wang [9], we consider the more general equation $\left(E_{\lambda}\right)$, the existence of least energy solutions for equation $\left(E_{\lambda}\right)$ with a potential well int $a^{-1}(0)$ for $\lambda$ large is proved under the conditions $\left(a_{1}\right),\left(a_{2}\right)$ and $\left(f_{1}\right),\left(f_{2}\right),\left(f_{3}\right)$.

The paper is organized as follows. In Section 2, we describe our main result (Theorem 2.1). In Section 3, we give some preliminaries that will be used for the proof of the main result. Finally, Theorem 2.1 will be proved in Section 4 .

Throughout this paper, we use the same $C$ to denote different universal constants.

\section{Main result}

Let $V_{\lambda}(x)=\lambda a(x)+1$. Formally, we define the following functional:

$$
J_{\lambda}(u)=\frac{1}{2} \int_{\mathbb{R}^{N}}\left(1+u^{2}\right)|\nabla u|^{2} d x+\frac{1}{2} \int_{\mathbb{R}^{N}} V_{\lambda}(x) u^{2} d x-\int_{\mathbb{R}^{N}} F(u) d x
$$

for $u \in X:=\left\{u \in H^{1}\left(\mathbb{R}^{N}\right) \mid \int_{\mathbb{R}^{N}} V_{\lambda}(x) u^{2}<\infty\right\}$. Note that under our assumptions, the functional $J_{\lambda}$ is not well defined on $X$.

We follow the idea of [9] and make the following change of variable.

Let $v=h(u)=\frac{1}{2} u \sqrt{1+u^{2}}+\frac{1}{2} \ln \left(u+\sqrt{1+u^{2}}\right)$, then $d v=\sqrt{1+u^{2}} d u$. Moreover, $h(u)$ satisfies

$$
h(u) \sim \begin{cases}u, & |u| \ll 1, \\ \frac{1}{2} u|u|, & |u| \gg 1 .\end{cases}
$$

Since $h^{\prime}(u)=\sqrt{1+u^{2}}>0, h(u)$ is strictly monotone and hence has an inverse function denoted by $u=g(v)$. Obviously,

$$
g(v) \sim\left\{\begin{array}{ll}
v, & |v| \ll 1, \\
\sqrt{\frac{2}{|v|}} v, & |v| \gg 1,
\end{array} \quad g^{\prime}(v)=\frac{1}{\sqrt{1+g^{2}(v)}} .\right.
$$

Let $G(v)=g^{2}(v)$. Then it holds that

$$
G(v)=g^{2}(v) \sim \begin{cases}v^{2}, & |v| \ll 1, \\ 2|v|, & |v| \gg 1\end{cases}
$$

and $G(v)$ is convex. Moreover, there exists $C_{0}>0$ such that $G(2 v) \leq C_{0} G(v)$,

$$
G^{\prime}(v)=\frac{2 g(v)}{\sqrt{1+g^{2}(v)}}, \quad G^{\prime \prime}(v)=\frac{2}{\left(1+g^{2}(v)\right)^{2}}>0 .
$$

Now we introduce the Orlicz space (see [26])

$$
E_{G}^{\lambda}=\left\{v \mid \int_{\mathbb{R}^{N}} V_{\lambda} G(v) d x<+\infty\right\}
$$

equipped with the norm

$$
|v|_{G}^{\lambda}:=\inf _{\xi>0} \xi\left(1+\int_{\mathbb{R}^{N}} V_{\lambda} G\left(\xi^{-1} v\right) d x\right) .
$$

Then $E_{G}^{\lambda}$ is a Banach space. 
Let

$$
H_{G}^{\lambda}:=\left\{\left.v \in E_{G}^{\lambda}\left|\int_{\mathbb{R}^{N}}\right| \nabla v\right|^{2} d x<+\infty\right\}
$$

equipped with the norm

$$
\|v\|_{\lambda}=\|\nabla v\|_{L^{2}}+|v|_{G}^{\lambda}
$$

Using the change of variable, we define the functional $\Phi_{\lambda}$ on $H_{G}^{\lambda}$ by

$$
\Phi_{\lambda}(v)=\frac{1}{2} \int_{\mathbb{R}^{N}}|\nabla v|^{2} d x+\frac{1}{2} \int_{\mathbb{R}^{N}} V_{\lambda} g^{2}(v) d x-\int_{\mathbb{R}^{N}} F\left(g\left(v_{+}\right)\right) d x,
$$

where $v_{+}=\max \{v, 0\}$ is the positive part of $v$.

Let

$$
\left.N_{\lambda}:=\left\{v \in H_{G}^{\lambda} \backslash\{0\}|| \Phi_{\lambda}^{\prime}(v), v\right\rangle=0\right\}
$$

be the Nehari manifold and let

$$
c_{\lambda}:=\inf _{v \in N_{\lambda}} \Phi_{\lambda}(v)
$$

be the infimum of $\Phi_{\lambda}$ on the Nehari manifold $N_{\lambda}$, where $\Phi_{\lambda}^{\prime}(v)$ is the Gateaux derivative (see Proposition 3.3).

We say that $u_{\lambda}=g\left(v_{\lambda}\right)$ is a least energy solution of $\left(E_{\lambda}\right)$ if $v_{\lambda} \in N_{\lambda}$ such that $c_{\lambda}$ is achieved.

Note that under our assumptions, for $\lambda$ large enough, the following Dirichlet problem is a kind of a 'limit' problem:

$$
\begin{cases}-\Delta u+u-\frac{1}{2}\left(\Delta|u|^{2}\right) u=f(u), & x \in \Omega, \\ u>0, & x \in \Omega, \\ u=0, & x \in \partial \Omega,\end{cases}
$$

where $\Omega:=\operatorname{int} a^{-1}(0)$.

Similar to the definition of the least energy solution of $\left(E_{\lambda}\right)$, we can define the least energy solution of $(D)$ which will be given in Section 4.

Our main result is as follows.

Theorem 2.1 Assume that $\left(a_{1}\right),\left(a_{2}\right)$ and $\left(f_{1}\right),\left(f_{2}\right),\left(f_{3}\right)$ are satisfied. Then for $\lambda$ large, $c_{\lambda}$ is achieved by a critical point $v_{\lambda}$ of $\Phi_{\lambda}$ such that $u_{\lambda}=g\left(v_{\lambda}\right)$ is a least energy solution of $\left(E_{\lambda}\right)$. Furthermore, for any sequence $\lambda_{n} \rightarrow \infty,\left\{v_{\lambda_{n}}\right\}$ has a subsequence converging to $v$ such that $u=g(v)$ is a least energy solution of $(D)$.

\section{Preliminaries}

In order to obtain the compactness of the functional $\Phi_{\lambda}$, we recall the following Lemmas 3.1 and 3.2 which can be found in [13]. 
Lemma 3.1 There exist two constants $C_{1}>0, C_{2}>0$ such that

$$
C_{1} \min \left\{\|v\|_{\lambda},\|v\|_{\lambda}^{2}\right\} \leq \int_{\mathbb{R}^{N}}|\nabla v|^{2} d x+\int_{\mathbb{R}^{N}} V_{\lambda}(x) g^{2}(v) d x \leq C_{2} \max \left\{\|v\|_{\lambda},\|v\|_{\lambda}^{2}\right\}
$$

for any $v \in H_{G}^{\lambda}$.

Lemma 3.2 The map: $v \rightarrow g(v)$ from $H_{G}^{\lambda}$ into $L^{q}\left(\mathbb{R}^{N}\right)$ is continuous for $2 \leq q \leq 2 \cdot 2^{*}$.

Now we consider the functional $\Phi_{\lambda}$ defined on $H_{G}^{\lambda}$ by (2.2), the following Proposition 3.3 is due to [9].

\section{Proposition 3.3}

(i) $\Phi_{\lambda}$ is well defined on $H_{G}^{\lambda}$;

(ii) $\Phi_{\lambda}$ is continuous in $H_{G}^{\lambda}$;

(iii) $\Phi_{\lambda}$ is Gateaux differentiable, the Gateaux derivative $\Phi_{\lambda}^{\prime}(v)$ for $v \in H_{G}^{\lambda}$ is a linear functional and $\Phi_{\lambda}^{\prime}(v)$ is continuous in $v$ in the strong-weak topology, that is, if $v_{n} \rightarrow v$ strongly in $H_{G}^{\lambda}$, then $\Phi_{\lambda}^{\prime}\left(v_{n}\right) \rightarrow \Phi_{\lambda}^{\prime}(v)$ weakly. Moreover, the Gateaux derivative $\Phi_{\lambda}^{\prime}(v)$ has the form

$$
\begin{aligned}
\left\langle\Phi_{\lambda}^{\prime}(v), w\right\rangle= & \int_{\mathbb{R}^{N}} \nabla v \nabla w d x+\int_{\mathbb{R}^{N}} V_{\lambda}(x) g(v) g^{\prime}(v) w d x \\
& -\int_{\mathbb{R}^{N}} f\left(g\left(v_{+}\right)\right) g^{\prime}\left(v_{+}\right) w d x .
\end{aligned}
$$

Recall that $\left\{v_{n}\right\} \subset H_{G}^{\lambda}$ is called a Palais-Smale sequence ((PS) $)_{c}$ sequence in short) for $\Phi_{\lambda}$ if $\Phi_{\lambda}\left(v_{n}\right) \rightarrow c$ and $\Phi_{\lambda}^{\prime}\left(v_{n}\right) \rightarrow 0$ in $\left(H_{G}^{\lambda}\right)^{*}$, the dual space of $H_{G}^{\lambda}$. We say that the functional $\Phi_{\lambda}$ satisfies the (PS) ${ }_{c}$ condition if any of (PS) ${ }_{c}$ sequence (up to a subsequence, if necessary) $\left\{v_{n}\right\}$ converges strongly in $H_{G}^{\lambda}$.

Lemma 3.4 Any of $(P S)_{c}$ sequence $\left\{v_{n}\right\}$ for $\Phi_{\lambda}$ is bounded.

Proof Suppose that $\left\{v_{n}\right\}$ is a (PS) $)_{c}$ sequence of $\Phi_{\lambda}$. We have $\Phi_{\lambda}\left(v_{n}\right) \rightarrow c \in \mathbb{R}$ and $\Phi_{\lambda}^{\prime}\left(v_{n}\right) \rightarrow$ 0 in the space $\left(H_{G}^{\lambda}\right)^{*}$.

Taking $w_{n}=\frac{g\left(v_{n}\right)}{g^{\prime}\left(v_{n}\right)}$, then $\left|\nabla w_{n}\right|=\left(1+\frac{g^{2}\left(v_{n}\right)}{1+g^{2}\left(v_{n}\right)}\right)\left|\nabla v_{n}\right| \leq 2\left|\nabla v_{n}\right|$, we have $\left\|w_{n}\right\|_{\lambda} \leq C\left\|v_{n}\right\|_{\lambda}$, thus

$$
\begin{aligned}
o\left(\left\|v_{n}\right\|_{\lambda}\right)= & \left\langle\Phi_{\lambda}^{\prime}\left(v_{n}\right), \frac{g\left(v_{n}\right)}{g^{\prime}\left(v_{n}\right)}\right\rangle \\
= & \int_{\mathbb{R}^{N}} \nabla v_{n} \nabla w_{n} d x+\int_{\mathbb{R}^{N}} V_{\lambda} g\left(v_{n}\right) g^{\prime}\left(v_{n}\right) w_{n} d x-\int_{\mathbb{R}^{N}} f\left(g\left(\left(v_{n}\right)_{+}\right)\right) g^{\prime}\left(\left(v_{n}\right)_{+}\right) w_{n} d x \\
= & \int_{\mathbb{R}^{N}}\left(1+\frac{g^{2}\left(v_{n}\right)}{1+g^{2}\left(v_{n}\right)}\right)\left|\nabla v_{n}\right|^{2} d x+\int_{\mathbb{R}^{N}} V_{\lambda} g^{2}\left(v_{n}\right) d x \\
& -\int_{\mathbb{R}^{N}} f\left(g\left(\left(v_{n}\right)_{+}\right)\right) g\left(\left(v_{n}\right)_{+}\right) d x
\end{aligned}
$$

and

$$
\Phi_{\lambda}\left(v_{n}\right)=\frac{1}{2} \int_{\mathbb{R}^{N}}\left|\nabla v_{n}\right|^{2} d x+\frac{1}{2} \int_{\mathbb{R}^{N}} V_{\lambda} g^{2}\left(v_{n}\right) d x-\int_{\mathbb{R}^{N}} F\left(g\left(\left(v_{n}\right)_{+}\right)\right) d x=c+o(1) .
$$


Taking (3.4) $-\frac{1}{\theta}(3.3)$ yields

$$
\begin{aligned}
\int_{\mathbb{R}^{N}} & \left(\frac{1}{2}-\frac{1}{\theta}\left(1+\frac{g^{2}\left(v_{n}\right)}{1+g^{2}\left(v_{n}\right)}\right)\right)\left|\nabla v_{n}\right|^{2} d x+\left(\frac{1}{2}-\frac{1}{\theta}\right) \int_{\mathbb{R}^{N}} V_{\lambda} g^{2}\left(v_{n}\right) d x \\
+ & \frac{1}{\theta} \int_{\mathbb{R}^{N}}\left(f\left(g\left(\left(v_{n}\right)_{+}\right)\right) g\left(\left(v_{n}\right)_{+}\right)-\theta F\left(g\left(\left(v_{n}\right)_{+}\right)\right)\right) d x=c+o(1)+o\left(\left\|v_{n}\right\|_{\lambda}\right) .
\end{aligned}
$$

Note that

$$
\begin{aligned}
& \frac{1}{2}-\frac{1}{\theta}\left(1+\frac{g^{2}\left(v_{n}\right)}{1+g^{2}\left(v_{n}\right)}\right)>\frac{1}{2}-\frac{2}{\theta}=\frac{\theta-4}{2 \theta}, \\
& \frac{1}{2}-\frac{1}{\theta}=\frac{\theta-2}{2 \theta}, \quad f(u) \cdot u>\theta F(u), \quad \theta>4,
\end{aligned}
$$

we have

$$
\int_{\mathbb{R}^{N}}\left|\nabla v_{n}\right|^{2} d x+\int_{\mathbb{R}^{N}} V_{\lambda} g^{2}\left(v_{n}\right) d x \leq \frac{2 \theta}{\theta-4}\left(c+o(1)+o\left(\left\|v_{n}\right\|_{\lambda}\right)\right) .
$$

It follows from Lemma 3.1 that

$$
C_{1} \min \left\{\|v\|_{\lambda},\|v\|_{\lambda}^{2}\right\} \leq \frac{2 \theta}{\theta-4}\left(c+o(1)+o\left(\left\|v_{n}\right\|_{\lambda}\right)\right),
$$

thus $\left\{v_{n}\right\}$ is bounded in $H_{G}^{\lambda}$.

Let $K_{\lambda}=\left\{v \in H_{G}^{\lambda} \mid \Phi_{\lambda}^{\prime}(v)=0\right\}$ be the critical set of $\Phi_{\lambda}$. Suppose $v \in K_{\lambda}$, then it is easy to check that either $v>0$ or $v \equiv 0$ in $\mathbb{R}^{N}$ by the definition of $\Phi_{\lambda}$ and the strong maximum principle.

Lemma 3.5 There exists $0<\sigma<1$ which is independent of $\lambda$ such that $\|v\|_{\lambda} \geq\|v\|_{1}>\sigma$ for all $\nu \in K_{\lambda} \backslash\{0\}$ and $\lambda \geq 1$.

Proof Assume that $\|v\|_{\lambda} \leq 1$ for any $v \in K_{\lambda} \backslash\{0\}$ (otherwise, the conclusion is true). From $\left(f_{1}\right),\left(f_{2}\right)$, we see that for any $\varepsilon>0$, there is a constant $C_{\varepsilon}>0$ such that $f(x, u) \leq \varepsilon u+C_{\varepsilon} u^{p}$ for $u>0$. We have

$$
\begin{aligned}
0 & =\left\langle\Phi_{\lambda}^{\prime}(v), \frac{g(v)}{g^{\prime}(v)}\right\rangle \\
& =\int_{\mathbb{R}^{N}}\left(\left(1+\frac{g^{2}(v)}{1+g^{2}(v)}\right)|\nabla v|^{2}+V_{\lambda} g^{2}(v)\right) d x-\int_{\mathbb{R}^{N}} f\left(g\left(v_{+}\right)\right) g\left(v_{+}\right) d x \\
& \geq \int_{\mathbb{R}^{N}}\left(|\nabla v|^{2}+V_{\lambda} g^{2}(v)\right) d x-\int_{\mathbb{R}^{N}} f\left(g\left(v_{+}\right)\right) g\left(v_{+}\right) d x \\
& \geq \int_{\mathbb{R}^{N}}\left(|\nabla v|^{2}+V_{\lambda} g^{2}(v)\right) d x-\int_{\mathbb{R}^{N}}\left(\varepsilon g^{2}\left(v_{+}\right)+C_{\varepsilon} g^{p+1}\left(v_{+}\right)\right) d x \\
& \geq \frac{1}{2} \int_{\mathbb{R}^{N}}\left(|\nabla v|^{2}+V_{\lambda} g^{2}(v)\right) d x-C_{\varepsilon} \int_{\mathbb{R}^{N}} g^{p+1}\left(v_{+}\right) d x \\
& \geq C_{1} \min \left\{\|v\|_{\lambda},\|v\|_{\lambda}^{2}\right\}-C_{2}\left(\max \left\{\|v\|_{\lambda},\|v\|_{\lambda}^{2}\right\}\right)^{\frac{p+1}{2}} \\
& =C_{1}\|v\|_{\lambda}^{2}-C_{2}\|v\|_{\lambda}^{\frac{p+1}{2}},
\end{aligned}
$$

and we can easily deduce the desired result. 
Lemma 3.6 There exists a positive constant $c_{0}>0$ such that

$$
\limsup _{n \rightarrow \infty}\left\|v_{n}\right\|_{\lambda} \leq \max \left\{\frac{2 \theta}{(\theta-4) C_{1}} c, \sqrt{\frac{2 \theta}{(\theta-4) C_{1}}} c\right\}
$$

and either $c \geq c_{0}$ or $c=0$ if $\left\{v_{n}\right\}$ is a $(P S)_{c}$ sequence for $\Phi_{\lambda}$, where $C_{1}$ is the constant in Lemma 3.1 .

Proof Since $\left\{v_{n}\right\}$ is a (PS) $)_{c}$ sequence, we have

$$
\begin{aligned}
\Phi_{\lambda}\left(v_{n}\right) & -\frac{1}{\theta}\left\langle\Phi_{\lambda}^{\prime}\left(v_{n}\right), \frac{g\left(v_{n}\right)}{g^{\prime}\left(v_{n}\right)}\right\rangle \\
= & \int_{\mathbb{R}^{N}}\left(\frac{1}{2}-\frac{1}{\theta}\left(1+\frac{g^{2}\left(v_{n}\right)}{1+g^{2}\left(v_{n}\right)}\right)\right)\left|\nabla v_{n}\right|^{2} d x+\left(\frac{1}{2}-\frac{1}{\theta}\right) \int_{\mathbb{R}^{N}} V_{\lambda} g^{2}\left(v_{n}\right) d x \\
& +\frac{1}{\theta} \int_{\mathbb{R}^{N}}\left(f\left(g\left(\left(v_{n}\right)_{+}\right)\right) g\left(\left(v_{n}\right)_{+}\right)-\theta F\left(g\left(\left(v_{n}\right)_{+}\right)\right)\right) d x \\
= & c+o(1)+o\left(\left\|v_{n}\right\|_{\lambda}\right) .
\end{aligned}
$$

It follows from (3.5) that

$$
\limsup _{n \rightarrow \infty}\left\|v_{n}\right\|_{\lambda} \leq \max \left\{\frac{2 \theta}{(\theta-4) C_{1}} c, \sqrt{\frac{2 \theta}{(\theta-4) C_{1}}} c\right\} .
$$

On the other hand, for $\left\|v_{n}\right\|_{\lambda} \leq 1$, we have

$$
\begin{aligned}
o\left(\left\|v_{n}\right\|_{\lambda}\right) & =\left\langle\Phi_{\lambda}^{\prime}\left(v_{n}\right), \frac{g\left(v_{n}\right)}{g^{\prime}\left(v_{n}\right)}\right\rangle \\
& \geq C_{1} \min \left\{\left\|v_{n}\right\|_{\lambda},\left\|v_{n}\right\|_{\lambda}^{2}\right\}-C_{2}\left(\max \left\{\left\|v_{n}\right\|_{\lambda},\left\|v_{n}\right\|_{\lambda}^{2}\right\}\right)^{\frac{p+1}{2}} \\
& =C_{1}\left\|v_{n}\right\|_{\lambda}^{2}-C_{2}\left\|v_{n}\right\|_{\lambda}^{\frac{p+1}{2}} .
\end{aligned}
$$

Thus, there exists $\sigma_{1}>0\left(\sigma_{1}<1\right)$ such that

$$
\left\langle\Phi_{\lambda}^{\prime}\left(v_{n}\right), \frac{g\left(v_{n}\right)}{g^{\prime}\left(v_{n}\right)}\right\rangle \geq \frac{1}{4} C_{1}\left\|v_{n}\right\|_{\lambda}^{2} \quad \text { for }\left\|v_{n}\right\|_{\lambda} \leq \sigma_{1} .
$$

Taking $c_{0}=\frac{\sigma_{1}}{\max \left\{\frac{2 \theta}{(\theta-4) C_{1}}, \sqrt{\frac{2 \theta}{(\theta-4) C_{1}}}\right\}}$, then we have

$$
\max \left\{\frac{2 \theta}{(\theta-4) C_{1}} c, \sqrt{\frac{2 \theta}{(\theta-4) C_{1}}} c\right\}<\sigma_{1}<1
$$

if $c<c_{0}$. It follows from (3.6) and (3.7) that

$$
\frac{1}{4}\left\|v_{n}\right\|_{\lambda}^{2} \leq o\left(\left\|v_{n}\right\|_{\lambda}\right)
$$

hence, $\left\|v_{n}\right\|_{\lambda} \rightarrow 0$ and $c=0$. Therefore, we have proved that there exists a constant $c_{0}$ such that either $c \geq c_{0}$ or $c=0$. 
Proposition 3.7 Let $M>0$ be a constant. Then for any $\varepsilon>0$, there exist $\Lambda_{\varepsilon}>0, R_{\varepsilon}>0$ such that

$$
\limsup _{n \rightarrow \infty} \int_{B_{R_{\varepsilon}}^{c}}\left(\frac{1}{2} f\left(g\left(\left(v_{n}\right)_{+}\right)\right) g\left(\left(v_{n}\right)_{+}\right)-F\left(g\left(\left(v_{n}\right)_{+}\right)\right)\right) d x \leq \varepsilon
$$

if $\left\{v_{n}\right\}$ is a $(P S)_{c}$ sequence of $\Phi_{\lambda}$ with $\lambda>\Lambda_{\varepsilon}, c \leq M$, where $B_{R_{\varepsilon}}^{c}=\left\{x \in \mathbb{R}^{N}|| x \mid \geq R_{\varepsilon}\right\}$.

Proof For all $R>0$, let

$$
\begin{aligned}
& A(R):=\left\{x \in \mathbb{R}^{N}|| x \mid \geq R, a(x) \geq M_{0}\right\}, \\
& B(R):=\left\{x \in \mathbb{R}^{N}|| x \mid \geq R, a(x) \leq M_{0}\right\} .
\end{aligned}
$$

We have

$$
\begin{aligned}
\int_{A(R)} g^{2}\left(v_{n}\right) d x & \leq \frac{1}{\lambda M_{0}+1} \int_{A(R)}(\lambda a(x)+1) g^{2}\left(v_{n}\right) d x \\
& \leq \frac{1}{\lambda M_{0}+1} \int_{A(R)}\left(\left|\nabla v_{n}\right|^{2}+(\lambda a(x)+1) g^{2}\left(v_{n}\right)\right) d x \\
& \leq \frac{1}{\lambda M_{0}+1}\left(\frac{2 \theta}{\theta-4} c+o\left(\left\|v_{n}\right\|_{\lambda}\right)\right) \\
& =\frac{1}{\lambda M_{0}+1}\left(\frac{2 \theta}{\theta-4} c+o(1)\right) \\
& \rightarrow 0 \quad(\lambda \rightarrow \infty) .
\end{aligned}
$$

On the other hand, by the Hölder inequality and interpolation inequality, we have

$$
\begin{aligned}
\int_{B(R)} g^{2}\left(v_{n}\right) d x & \leq\left(\int_{B(R)}\left(g^{2}\left(v_{n}\right)\right)^{q} d x\right)^{\frac{1}{q}}\left(\int_{B(R)} 1 d x\right)^{\frac{q-1}{q}} \quad\left(1<q<2^{*}\right) \\
& \leq \mu(B(R))^{\frac{q-1}{q}}\left(\int_{\mathbb{R}^{N}} g^{2}\left(v_{n}\right) d x\right)^{\beta}\left(\int_{\mathbb{R}^{N}} g^{2 \cdot 2^{*}}\left(v_{n}\right) d x\right)^{\frac{1-\beta}{2^{*}}} \quad(0<\beta<1) \\
& \leq C \mu(B(R))^{\frac{q-1}{q}}\left(\max \left\{\left\|v_{n}\right\|_{\lambda},\left\|v_{n}\right\|_{\lambda}^{2}\right\}\right)^{\beta}\left\|v_{n}\right\|_{\lambda}^{1-\beta} \\
& \leq C \mu(B(R))^{\frac{q-1}{q}} \quad\left(\left\|v_{n}\right\|_{\lambda} \text { is bounded }\right) . \\
& \rightarrow 0 \quad(R \rightarrow \infty) .
\end{aligned}
$$

By using the Gagliardo-Nirenberg inequality, we obtain

$$
\begin{aligned}
& \int_{B_{R_{\varepsilon}}^{c}}\left(\frac{1}{2} f\left(g\left(\left(v_{n}\right)_{+}\right)\right) g\left(\left(v_{n}\right)_{+}\right)-F\left(g\left(\left(v_{n}\right)_{+}\right)\right)\right) d x \\
& \quad \leq \int_{B_{R_{\varepsilon}}^{c}}\left(\frac{1}{2} f\left(g\left(\left(v_{n}\right)_{+}\right)\right) g\left(\left(v_{n}\right)_{+}\right)-C\left(g\left(\left(v_{n}\right)_{+}\right)\right)^{\theta}\right) d x \quad\left(\text { by }\left(f_{3}\right)\right) \\
& \quad \leq C \int_{B_{R}^{c}}\left|g\left(v_{n}\right)\right|^{p+1} d x
\end{aligned}
$$




$$
\begin{aligned}
& \leq C\left(\int_{B_{R}^{c}}\left|\nabla g\left(v_{n}\right)\right|^{2} d x\right)^{\frac{(p+1) \beta}{2}}\left(\int_{B_{R}^{c}} g^{2}\left(v_{n}\right) d x\right)^{\frac{(p+1)(1-\beta)}{2}} \quad\left(\beta=\frac{N(p-1)}{2(p+1)}\right) \\
& \leq C\left\|v_{n}\right\|_{\lambda}^{(p+1) \beta}\left(\int_{A(R)} g^{2}\left(v_{n}\right) d x+\int_{B(R)} g^{2}\left(v_{n}\right) d x\right)^{\frac{(p+1)(1-\beta)}{2}} \\
& \leq C\left(\int_{A(R)} g^{2}\left(v_{n}\right) d x+\int_{B(R)} g^{2}\left(v_{n}\right) d x\right)^{\frac{(p+1)(1-\beta)}{2}} \quad\left(\left\|v_{n}\right\|_{\lambda} \text { is bounded }\right) .
\end{aligned}
$$

Let $\lambda$ and $R$ be large enough, from (3.8) and (3.9), we get the desired result.

Lemma $3.8 c_{\lambda}=\inf _{N_{\lambda}} \Phi_{\lambda}(v)$ is achieved by some $v>0$.

Proof By the definition of $c_{\lambda}$ and the Ekeland variational principle, there exists a (PS) sequence $\left\{v_{n}\right\}$, by Lemma 3.4, we know that $\left\{v_{n}\right\}$ is bounded. Hence (up to a subsequence) we have $v_{n} \rightarrow v$ in $L^{2}, \nabla v_{n} \rightarrow \nabla v$ in $L^{2}, v_{n} \rightarrow v$ a.e. in $\mathbb{R}^{N}, g\left(v_{n}\right) \rightarrow g(v)$ in $L^{q}$ for $2 \leq q \leq$ $2 \cdot 2^{*}$.

It is sufficient to prove that $v \neq 0$ and $v \in N_{\lambda}$. In fact,

$$
\begin{aligned}
& \Phi_{\lambda}\left(v_{n}\right)-\frac{1}{2}\left\langle\Phi_{\lambda}^{\prime}\left(v_{n}\right), \frac{g\left(v_{n}\right)}{g^{\prime}\left(v_{n}\right)}\right\rangle \\
& =-\frac{1}{2} \int_{\mathbb{R}^{N}} \frac{g^{2}\left(v_{n}\right)}{1+g^{2}\left(v_{n}\right)}\left|\nabla v_{n}\right|^{2} d x+\int_{\mathbb{R}^{N}}\left(\frac{1}{2} f\left(g\left(\left(v_{n}\right)_{+}\right)\right) g\left(\left(v_{n}\right)_{+}\right)-F\left(g\left(\left(v_{n}\right)_{+}\right)\right)\right) d x \\
& =c+o(1)+o\left(\left\|v_{n}\right\|_{\lambda}\right)
\end{aligned}
$$

it follows that

$$
\int_{\mathbb{R}^{N}}\left(\frac{1}{2} f\left(g\left(\left(v_{n}\right)_{+}\right)\right) g\left(\left(v_{n}\right)_{+}\right)-F\left(g\left(\left(v_{n}\right)_{+}\right)\right)\right) \geq c+o(1)+o\left(\left\|v_{n}\right\|_{\lambda}\right) .
$$

Let $\varepsilon_{0}=\frac{1}{4} c$, since $g\left(v_{n}\right) \rightarrow g(v)$ strongly in $L^{p+1}\left(B_{R}\right)$ for $R>0$, by Proposition 3.7, there exist $\Lambda_{0}>0, R_{0}>0$ such that for $\lambda \geq \Lambda_{0}, R \geq R_{0}$,

$$
\int_{B_{R}^{c}}\left|\left(\frac{1}{2} f\left(g\left(\left(v_{n}\right)_{+}\right)\right) g\left(\left(v_{n}\right)_{+}\right)-F\left(g\left(\left(v_{n}\right)_{+}\right)\right)\right)-\left(\frac{1}{2} f\left(g\left(v_{+}\right)\right) g\left(v_{+}\right)-F\left(g\left(v_{+}\right)\right)\right)\right| d x<\varepsilon_{0},
$$

thus

$$
\int_{B_{R}}\left(\frac{1}{2} f\left(g\left(v_{+}\right)\right) g\left(v_{+}\right)-F\left(g\left(v_{+}\right)\right)\right) d x \geq \frac{1}{2} c>0 .
$$

Hence $v \neq 0$.

Now we prove $v \in N_{\lambda}$. Indeed, since $\left\{v_{n}\right\}$ is a (PS) $)_{c}$ sequence, we have

$$
\begin{aligned}
& \int_{\mathbb{R}^{N}} \nabla v_{n} \nabla v d x+\int_{\mathbb{R}^{N}} V_{\lambda} g\left(v_{n}\right) g^{\prime}\left(v_{n}\right) v d x \\
& \quad=\int_{\mathbb{R}^{N}} f\left(g\left(\left(v_{n}\right)_{+}\right)\right) g^{\prime}\left(\left(v_{n}\right)_{+}\right) v d x+o\left(\left\|v_{n}\right\|_{\lambda}\right),
\end{aligned}
$$

where $o\left(\left\|v_{n}\right\|_{\lambda}\right) \rightarrow 0$ as $n \rightarrow \infty$ 
Let $l_{n}:=\frac{g\left(v_{n}\right)}{\sqrt{1+g^{2}\left(v_{n}\right)}}$, then $\left\{l_{n}\right\}$ is bounded in $L^{q}\left(\mathbb{R}^{N}\right)$ for $2 \leq q \leq 2 \cdot 2^{*}$, by the continuity of $g$, we have, up to a subsequence, $l_{n} \rightarrow l=\frac{g(v)}{\sqrt{1+g^{2}(v)}}$ in $L^{q}\left(\mathbb{R}^{N}\right)$.

Similarly, we have $s_{n}:=\frac{f\left(g\left(v_{n}\right)\right)}{\sqrt{1+g^{2}\left(v_{n}\right)}} \leq f\left(g\left(v_{n}\right)\right)$ is bounded in $L^{\frac{p+1}{p}}\left(\mathbb{R}^{N}\right)$. Again, by the continuity of $g$, we have $s_{n} \rightarrow s=\frac{f(g(v))}{\sqrt{1+g^{2}(v)}}$ in $L^{\frac{p+1}{p}}\left(\mathbb{R}^{N}\right)$. Passing to the limits in (3.11), we get

$$
\int_{\mathbb{R}^{N}}|\nabla v|^{2} d x+\int_{\mathbb{R}^{N}} V_{\lambda} g(v) g^{\prime}(v) v d x=\int_{\mathbb{R}^{N}} f\left(g\left(v_{+}\right)\right) g^{\prime}\left(v_{+}\right) v d x,
$$

which is equivalent to $\left\langle\Phi_{\lambda}^{\prime}(v), v\right\rangle=0$, that is, $v \in N_{\lambda}$.

\section{Proof of the main result}

Consider the following quasilinear Schrödinger equation in $\Omega \subset \mathbb{R}^{N}(N \geq 3)$ :

$$
\begin{cases}-\Delta u+u-\frac{1}{2}\left(\Delta|u|^{2}\right) u=f(u), & x \in \Omega, \\ u>0, & x \in \Omega, \\ u=0, & x \in \partial \Omega .\end{cases}
$$

We have the same change of variables and the same notation as in the previous sections. Define the corresponding Orlicz space $E_{G}(\Omega)$ by

$$
E_{G}(\Omega)=\left\{v \mid \int_{\Omega} g^{2}(v) d x<+\infty\right\}
$$

with the norm

$$
|v|_{G(\Omega)}:=\inf _{\xi>0} \xi\left(1+\int_{\Omega} G\left(\xi^{-1} v\right) d x\right)
$$

The space $H_{G}(\Omega)$ is defined by

$$
H_{G}(\Omega):=\left\{\left.v\left|\int_{\Omega}\right| \nabla v\right|^{2} d x<+\infty, \int_{\Omega} g^{2}(v) d x<+\infty\right\}
$$

with the norm

$$
\|v\|_{\Omega}=\|v\|_{L^{2}}+|v|_{G(\Omega)}
$$

The following Lemma 4.1 is a counterpart of Lemma 3.1.

Lemma 4.1 There exist two constants $C_{1}>0, C_{2}>0$ such that

$$
C_{1} \min \left\{\|v\|_{\Omega},\|v\|_{\Omega}^{2}\right\} \leq \int_{\Omega}|\nabla v|^{2} d x+\int_{\Omega} g^{2}(v) d x \leq C_{2} \max \left\{\|v\|_{\Omega},\|v\|_{\Omega}^{2}\right\}
$$

for any $v \in H_{G}(\Omega)$. 
We denote by $H_{G}^{0}(\Omega)$ the closure of $C_{0}^{\infty}(\Omega)$ in $H_{G}(\Omega)$. We define the functional $\Phi_{\Omega}$ on $H_{G}^{0}(\Omega)$ by

$$
\Phi_{\Omega}(v)=\frac{1}{2} \int_{\Omega}\left(|\nabla v|^{2}+g^{2}(v)\right) d x-\int_{\Omega} F\left(g\left(v_{+}\right)\right) d x,
$$

and we define the Nehari manifold $N_{\Omega}$ by

$$
\left.N_{\Omega}:=\left\{v \in H_{G}(\Omega) \backslash\{0\}|| \Phi_{\Omega}^{\prime}(v), v\right\rangle=0\right\} .
$$

Let

$$
c(\Omega)=\inf _{N_{\Omega}} \Phi_{\Omega}(v) .
$$

We recall that $u=g(v)$ is a least energy solution of $(D)$ if $v \in N_{\Omega}$ such that $c(\Omega)$ is achieved.

Lemma 4.2 Suppose $c_{\lambda}=\inf _{N_{\lambda}} \Phi_{\lambda}$. Then $\lim _{\lambda \rightarrow+\infty} c_{\lambda}=c(\Omega)$.

Proof It is easy to see that $c_{\lambda} \leq c(\Omega)$ for $\lambda \geq 1$. We claim that $c_{\lambda}$ is monotone increasing with respect to $\lambda$. In fact, for $\lambda_{1}<\lambda_{2}$, we assume that $c_{\lambda_{1}}, c_{\lambda_{2}}$ are achieved for $\nu_{\lambda_{1}}>0$, $\nu_{\lambda_{2}}>0$. Obviously,

$$
\int_{\mathbb{R}^{N}}\left|\nabla \nu_{\lambda_{2}}\right|^{2} d x+\int_{\mathbb{R}^{N}}\left(\lambda_{1} a+1\right) g\left(v_{\lambda_{2}}\right) g^{\prime}\left(\nu_{\lambda_{2}}\right) v_{\lambda_{2}} d x<\int_{\mathbb{R}^{N}} f\left(g\left(v_{\lambda_{2}}\right)\right) g^{\prime}\left(v_{\lambda_{2}}\right) v_{\lambda_{2}} d x .
$$

We first prove that there exists $0<\alpha<1$ such that $\alpha v_{\lambda_{2}} \in N_{\lambda_{1}}$. This is sufficient to prove that

$$
\begin{aligned}
& \alpha^{2} \int_{\mathbb{R}^{N}}\left|\nabla v_{\lambda_{2}}\right|^{2} d x+\int_{\mathbb{R}^{N}}\left(\lambda_{1} a+1\right) \frac{g\left(\alpha v_{\lambda_{2}}\right)}{\sqrt{1+g^{2}\left(\alpha v_{\lambda_{2}}\right)}} \alpha v_{\lambda_{2}} d x \\
& \quad=\int_{\mathbb{R}^{N}} f\left(g\left(\alpha v_{\lambda_{2}}\right)\right) g^{\prime}\left(\alpha v_{\lambda_{2}}\right) \alpha v_{\lambda_{2}} d x .
\end{aligned}
$$

That is,

$$
\alpha \int_{\mathbb{R}^{N}}\left|\nabla v_{\lambda_{2}}\right|^{2} d x+\int_{\mathbb{R}^{N}}\left(\lambda_{1} a+1\right) \frac{g\left(\alpha \nu_{\lambda_{2}}\right)}{\sqrt{1+g^{2}\left(\alpha v_{\lambda_{2}}\right)}} v_{\lambda_{2}} d x=\int_{\mathbb{R}^{N}} f\left(g\left(\alpha v_{\lambda_{2}}\right)\right) g^{\prime}\left(\alpha v_{\lambda_{2}}\right) v_{\lambda_{2}} d x .
$$

Let

$$
I(\alpha)=\int_{\mathbb{R}^{N}} \frac{\left(f\left(g\left(\alpha v_{\lambda_{2}}\right)\right)-\left(\lambda_{1} a+1\right) g\left(\alpha v_{\lambda_{2}}\right)\right) v_{\lambda_{2}}}{\alpha \sqrt{1+g^{2}\left(\alpha v_{\lambda_{2}}\right)}} d x
$$

Then by $\left(f_{1}\right)$, we can obtain $\lim _{\alpha \rightarrow 0} I(\alpha) \leq 0$ and

$$
0<\int_{\mathbb{R}^{N}}\left|\nabla v_{\lambda_{2}}\right|^{2} d x<I(1) .
$$

Hence, there exists $0<\alpha_{0}<1$ such that $\int_{\mathbb{R}^{N}}\left|\nabla v_{\lambda_{2}}\right|^{2} d x=I\left(\alpha_{0}\right)$, i.e., $\alpha_{0} v_{\lambda_{2}} \in N_{\lambda_{1}}$. Thus

$$
c_{\lambda_{1}} \leq \Phi_{\lambda_{1}}\left(\alpha_{0} v_{\lambda_{2}}\right)
$$


In the following, we will prove that

$$
\Phi_{\lambda_{1}}\left(\alpha_{0} v_{\lambda_{2}}\right) \leq \Phi_{\lambda_{2}}\left(v_{\lambda_{2}}\right)=c_{\lambda_{2}}
$$

In fact, we consider the function $\rho(\alpha)$ defined by

$$
\rho(\alpha)=\frac{\left(f\left(g\left(\alpha v_{\lambda_{2}}\right)\right)-\left(\lambda_{2} a+1\right) g\left(\alpha v_{\lambda_{2}}\right)\right) v_{\lambda_{2}}}{\alpha \sqrt{1+g^{2}\left(\alpha \nu_{\lambda_{2}}\right)}} .
$$

By $g(t) \sqrt{1+g^{2}(t)} \leq 2 t$ for $t \geq 0$, we have $\frac{g\left(\alpha \nu_{\lambda_{2}}\right)}{g^{\prime}\left(\alpha \nu_{\lambda_{2}}\right)}=g\left(\alpha v_{\lambda_{2}}\right) \sqrt{1+g^{2}\left(\alpha v_{\lambda_{2}}\right)} \leq 2 \alpha v_{\lambda_{2}} \leq(\theta-$ 2) $\alpha v_{\lambda_{2}}$. It follows that

$$
\frac{\alpha v_{\lambda_{2}} g\left(\alpha \nu_{\lambda_{2}}\right)}{g\left(\alpha v_{\lambda_{2}}\right) / g^{\prime}\left(\alpha v_{\lambda_{2}}\right)+\alpha v_{\lambda_{2}}} \geq \frac{\alpha v_{\lambda_{2}} g\left(\alpha v_{\lambda_{2}}\right)}{(\theta-2) \alpha v_{\lambda_{2}}+\alpha v_{\lambda_{2}}} .
$$

Obviously,

$$
f^{\prime}\left(g\left(\alpha \nu_{\lambda_{2}}\right)\right) g^{\prime}\left(\alpha v_{\lambda_{2}}\right) v_{\lambda_{2}}^{2} \alpha g\left(\alpha \nu_{\lambda_{2}}\right)-f\left(g\left(\alpha v_{\lambda_{2}}\right)\right) \nu_{\lambda_{2}}\left(g\left(\alpha v_{\lambda_{2}}\right)+\alpha g^{\prime}\left(\alpha \nu_{\lambda_{2}}\right) \nu_{\lambda_{2}}\right) \geq 0
$$

and hence it is easy to check that

$$
\begin{aligned}
& \frac{d}{d \alpha}\left(\frac{f\left(g\left(\alpha v_{\lambda_{2}}\right)\right) \nu_{\lambda_{2}}}{\alpha \sqrt{1+g^{2}\left(\alpha v_{\lambda_{2}}\right)}}\right) \\
& =\frac{d}{d \alpha}\left(\frac{g\left(\alpha v_{\lambda_{2}}\right)}{\sqrt{1+g^{2}\left(\alpha v_{\lambda_{2}}\right)}}\right) \frac{f\left(g\left(\alpha v_{\lambda_{2}}\right)\right) v_{\lambda_{2}}}{\alpha g\left(\alpha v_{\lambda_{2}}\right)}+\frac{d}{d \alpha}\left(\frac{f\left(g\left(\alpha v_{\lambda_{2}}\right)\right) v_{\lambda_{2}}}{\alpha g\left(\alpha v_{\lambda_{2}}\right)}\right) \frac{g\left(\alpha v_{\lambda_{2}}\right)}{\sqrt{1+g^{2}\left(\alpha v_{\lambda_{2}}\right)}} \\
& =\frac{v_{\lambda_{2}}^{2}}{\left(1+g^{2}\left(\alpha v_{\lambda_{2}}\right)\right)^{2}} \cdot \frac{f\left(g\left(\alpha \nu_{\lambda_{2}}\right)\right) \nu_{\lambda_{2}}}{\alpha g\left(\alpha v_{\lambda_{2}}\right)} \\
& +\frac{f^{\prime}\left(g\left(\alpha v_{\lambda_{2}}\right)\right) g^{\prime}\left(\alpha v_{\lambda_{2}}\right) v_{\lambda_{2}}^{2} \alpha g\left(\alpha v_{\lambda_{2}}\right)-f\left(g\left(\alpha v_{\lambda_{2}}\right)\right) v_{\lambda_{2}}\left(g\left(\alpha v_{\lambda_{2}}\right)+\alpha g^{\prime}\left(\alpha v_{\lambda_{2}}\right) v_{\lambda_{2}}\right)}{\alpha^{2} g^{2}\left(\alpha v_{\lambda_{2}}\right)} \\
& \times \frac{g\left(\alpha \nu_{\lambda_{2}}\right)}{\sqrt{1+g^{2}\left(\alpha \nu_{\lambda_{2}}\right)}} \\
& \geq \frac{f^{\prime}\left(g\left(\alpha v_{\lambda_{2}}\right)\right) g^{\prime}\left(\alpha v_{\lambda_{2}}\right) v_{\lambda_{2}}^{2} \alpha g\left(\alpha v_{\lambda_{2}}\right)-f\left(g\left(\alpha v_{\lambda_{2}}\right)\right) v_{\lambda_{2}}\left(g\left(\alpha v_{\lambda_{2}}\right)+\alpha g^{\prime}\left(\alpha v_{\lambda_{2}}\right) v_{\lambda_{2}}\right)}{\alpha^{2} g\left(\alpha v_{\lambda_{2}}\right) \sqrt{1+g^{2}\left(\alpha v_{\lambda_{2}}\right)}} \\
& \geq 0 \text {. }
\end{aligned}
$$

On the other hand,

$$
\begin{aligned}
& \frac{d}{d \alpha}\left(\left(\lambda_{2} a+1\right) \frac{g\left(\alpha v_{\lambda_{2}}\right) v_{\lambda_{2}}}{\alpha \sqrt{1+g^{2}\left(\alpha \nu_{\lambda_{2}}\right)}}\right) \\
& \quad=\left(\lambda_{2} a+1\right) \frac{\alpha v_{\lambda_{2}}^{2}-g\left(\alpha v_{\lambda_{2}}\right) \sqrt{1+g^{2}\left(\alpha \nu_{\lambda_{2}}\right)}\left(1+g^{2}\left(\alpha \nu_{\lambda_{2}}\right)\right)}{\alpha^{2}\left(1+g^{2}\left(\alpha \nu_{\lambda_{2}}\right)\right)^{2}} v_{\lambda_{2}},
\end{aligned}
$$

by $v=\frac{1}{2} g(v) \sqrt{1+g^{2}(v)}+\frac{1}{2} \ln \left(g(v)+\sqrt{1+g^{2}(v)}\right)$, it is easy to check that for any $t \geq 0$,

$$
t-g(t) \sqrt{1+g^{2}(t)}\left(1+g^{2}(t)\right) \leq 0,
$$


which implies

$$
\frac{d}{d \alpha}\left(\left(\lambda_{2} a+1\right) \frac{g\left(\alpha v_{\lambda_{2}}\right) v_{\lambda_{2}}}{\alpha \sqrt{1+g^{2}\left(\alpha v_{\lambda_{2}}\right)}}\right) \leq 0
$$

for any $\alpha>0$, thus we have proved that $\rho(\alpha)$ is monotone increasing for $\alpha>0$.

Now we consider the function $\gamma(\alpha)$ defined by

$$
\gamma(\alpha)=\frac{1}{2} \int_{\mathbb{R}^{N}}\left(\alpha^{2}\left|\nabla \nu_{\lambda_{2}}\right|^{2}+\left(\lambda_{2} a(x)+1\right) g^{2}\left(\alpha \nu_{\lambda_{2}}\right)\right) d x-\int_{\mathbb{R}^{N}} F\left(g\left(\alpha \nu_{\lambda_{2}}\right)\right) d x .
$$

Then

$$
\begin{aligned}
\frac{d \gamma(\alpha)}{d \alpha}= & \int_{\mathbb{R}^{N}}\left(\alpha\left|\nabla \nu_{\lambda_{2}}\right|^{2}+\left(\lambda_{2} a(x)+1\right) g\left(\alpha v_{\lambda_{2}}\right) g^{\prime}\left(\alpha v_{\lambda_{2}}\right) v_{\lambda_{2}}\right) d x \\
& -\int_{\mathbb{R}^{N}} f\left(g\left(\alpha v_{\lambda_{2}}\right)\right) g^{\prime}\left(\alpha v_{\lambda_{2}}\right) v_{\lambda_{2}} d x \\
= & \alpha\left(\int_{\mathbb{R}^{N}} f\left(g\left(v_{\lambda_{2}}\right)\right) g^{\prime}\left(v_{\lambda_{2}}\right) v_{\lambda_{2}} d x-\int_{\mathbb{R}^{N}}\left(\lambda_{2} a(x)+1\right) g\left(v_{\lambda_{2}}\right) g^{\prime}\left(v_{\lambda_{2}}\right) v_{\lambda_{2}} d x\right) \\
& +\left(\int_{\mathbb{R}^{N}}\left(\lambda_{2} a(x)+1\right) g\left(\alpha v_{\lambda_{2}}\right) g^{\prime}\left(\alpha v_{\lambda_{2}}\right) v_{\lambda_{2}} d x-\int_{\mathbb{R}^{N}} f\left(g\left(\alpha v_{\lambda_{2}}\right)\right) g^{\prime}\left(\alpha v_{\lambda_{2}}\right) v_{\lambda_{2}} d x\right) \\
= & \alpha \int_{\mathbb{R}^{N}} \frac{\left(f\left(g\left(v_{\lambda_{2}}\right)\right)-\left(\lambda_{2} a(x)+1\right) g\left(\nu_{\lambda_{2}}\right)\right) v_{\lambda_{2}}}{\sqrt{1+g^{2}\left(v_{\lambda_{2}}\right)}} d x \\
& -\alpha \int_{\mathbb{R}^{N}} \frac{\left(f\left(g\left(\alpha v_{\lambda_{2}}\right)\right)-\left(\lambda_{2} a(x)+1\right) g\left(\alpha v_{\lambda_{2}}\right)\right) v_{\lambda_{2}}}{\alpha \sqrt{1+g^{2}\left(\alpha \nu_{\lambda_{2}}\right)}} d x
\end{aligned}
$$

$\geq 0 \quad$ (by the monotonicity of $\rho(\alpha)$ )

for $0<\alpha<1$. Therefore, $\gamma(\alpha)$ is monotone increasing with respect to $\alpha<1$. Thus, we deduce that

$$
\begin{aligned}
c_{\lambda_{1}} & \leq \Phi_{\lambda_{1}}\left(\alpha v_{\lambda_{2}}\right) \\
& =\frac{1}{2} \int_{\mathbb{R}^{N}} \alpha^{2}\left|\nabla v_{\lambda_{2}}\right|^{2} d x+\int_{\mathbb{R}^{N}}\left(\lambda_{1} a(x)+1\right) g^{2}\left(\alpha v_{\lambda_{2}}\right) d x-\int_{\mathbb{R}^{N}} F\left(g\left(\alpha v_{\lambda_{2}}\right)\right) d x \\
& \leq \frac{1}{2} \int_{\mathbb{R}^{N}}\left|\nabla v_{\lambda_{2}}\right|^{2} d x+\int_{\mathbb{R}^{N}}\left(\lambda_{2} a(x)+1\right) g^{2}\left(v_{\lambda_{2}}\right) d x-\int_{\mathbb{R}^{N}} F\left(g\left(v_{\lambda_{2}}\right)\right) d x \\
& =\Phi_{\lambda_{2}}\left(v_{\lambda_{2}}\right)=c_{\lambda_{2}} .
\end{aligned}
$$

Assume that $\lim _{\lambda \rightarrow \infty} c_{\lambda}=k \leq c(\Omega)$. If $k<c(\Omega)$, then for any sequence $\left\{\lambda_{n}\right\}\left(\lambda_{n} \rightarrow+\infty\right)$, we have $c_{\lambda_{n}} \rightarrow k<c(\Omega)$.

We assume that $v_{n}$ is such that $c_{\lambda_{n}}$ is achieved, by Lemma 3.4, $\left\{v_{n}\right\}$ is bounded in $H_{G}^{\lambda_{n}}$. Since $\left\|v_{n}\right\|_{H_{G}^{0}} \leq\left\|v_{n}\right\|_{H_{G}^{\lambda_{n}}},\left\{v_{n}\right\}$ is bounded in $H_{G}^{0}$, as a result, we have $\nabla v_{n} \rightarrow \nabla v$ in $L^{2}\left(\mathbb{R}^{N}\right)$, $g\left(v_{n}\right) \rightarrow g(v)$ in $L_{\mathrm{loc}}^{q}\left(\mathbb{R}^{N}\right)$ for $2 \leq q \leq 2 \cdot 2^{*}, v_{n} \rightarrow v$ in $L^{q}\left(\mathbb{R}^{N}\right)$ for $2 \leq q \leq 2 \cdot 2^{*}, v_{n} \rightarrow v$ a.e. in $\mathbb{R}^{N}$.

We claim that $\left.v\right|_{\Omega^{c}}=0$, where $\Omega^{c}:=\left\{x \mid x \in \mathbb{R}^{N} \backslash \Omega\right\}$. Indeed, it is sufficient to prove $\left.g(v)\right|_{\Omega^{c}}=0$. If not, then there exists a compact subset $\Sigma \subset \Omega^{c}$ with $\operatorname{dist}\{\Sigma, \partial \Omega\}>0$ such 
that $\left.g(v)\right|_{\Sigma} \neq 0$ and

$$
\int_{\Sigma} g^{2}\left(v_{n}\right) d x \rightarrow \int_{\Sigma} g^{2}(v) d x>0
$$

Moreover, there exists $\varepsilon_{0}>0$ such that $a(x) \geq \varepsilon_{0}$ for any $x \in \Sigma$.

By the choice of $v_{n}$, we have

$$
\begin{aligned}
0 & =\left\langle\Phi_{\lambda_{n}}^{\prime}\left(v_{n}\right), \frac{g\left(v_{n}\right)}{g^{\prime}\left(v_{n}\right)}\right\rangle \\
& =\int_{\mathbb{R}^{N}}\left(1+\frac{g^{2}\left(v_{n}\right)}{1+g^{2}\left(v_{n}\right)}\right)\left|\nabla v_{n}\right|^{2} d x+\int_{\mathbb{R}^{N}} V_{\lambda_{n}} g^{2}\left(v_{n}\right) d x-\int_{\mathbb{R}^{N}} f\left(g\left(\left(v_{n}\right)_{+}\right)\right) g\left(\left(v_{n}\right)_{+}\right) d x,
\end{aligned}
$$

hence,

$$
\begin{aligned}
\Phi_{\lambda_{n}}\left(v_{n}\right)= & \frac{1}{2} \int_{\mathbb{R}^{N}}\left(\left|\nabla v_{n}\right|^{2}+V_{\lambda_{n}} g^{2}\left(v_{n}\right)\right) d x-\int_{\mathbb{R}^{N}} F\left(g\left(\left(v_{n}\right)_{+}\right)\right) d x \\
\geq & \frac{1}{2} \int_{\mathbb{R}^{N}}\left(\left|\nabla v_{n}\right|^{2}+V_{\lambda_{n}} g^{2}\left(v_{n}\right)\right) d x-\frac{1}{\theta} \int_{\mathbb{R}^{N}} f\left(g\left(\left(v_{n}\right)_{+}\right)\right) g\left(\left(v_{n}\right)_{+}\right) d x, \\
= & \frac{1}{2} \int_{\mathbb{R}^{N}}\left(\left|\nabla v_{n}\right|^{2}+V_{\lambda_{n}} g^{2}\left(v_{n}\right)\right) d x \\
& -\frac{1}{\theta} \int_{\mathbb{R}^{N}}\left(\left(1+\frac{g^{2}\left(v_{n}\right)}{1+g^{2}\left(v_{n}\right)}\right)\left|\nabla v_{n}\right|^{2}+V_{\lambda_{n}} g^{2}\left(v_{n}\right)\right) d x \\
= & \int_{\mathbb{R}^{N}}\left(\frac{1}{2}-\frac{1}{\theta}\left(1+\frac{g^{2}\left(v_{n}\right)}{1+g^{2}\left(v_{n}\right)}\right)\right)\left|\nabla v_{n}\right|^{2} d x+\left(\frac{1}{2}-\frac{1}{\theta}\right) \int_{\mathbb{R}^{N}} V_{\lambda_{n}} g^{2}\left(v_{n}\right) d x \\
\geq & \left(\frac{1}{2}-\frac{1}{\theta}\right) \int_{\Sigma}\left(\lambda_{n} \varepsilon_{0}+1\right) g^{2}\left(v_{n}\right) d x \\
\rightarrow & +\infty \quad(n \rightarrow \infty) .
\end{aligned}
$$

This contradiction shows that $\left.g(v)\right|_{\Omega^{c}}=0$ and so does $v$.

Now we show that

$$
g\left(v_{n}\right) \rightarrow g(v) \quad \text { in } L^{q}\left(\mathbb{R}^{N}\right) \text { for } 2<q<2^{*} .
$$

Suppose that (4.3) is not true, then by the concentration compactness principle of Lions (see [12]), there exist $\delta>0, \varrho>0$ and $x_{n} \in \mathbb{R}^{N}$ with $\left|x_{n}\right| \rightarrow+\infty$ such that

$$
\limsup _{n \rightarrow \infty} \int_{B_{\varrho}\left(x_{n}\right)}\left|g\left(v_{n}\right)-g(v)\right|^{2} \geq \delta>0 .
$$

On the other hand, by the choice of $\left\{v_{n}\right\}$, we have

$$
\begin{aligned}
\Phi_{\lambda_{n}}\left(v_{n}\right) & =\frac{1}{2} \int_{\mathbb{R}^{N}}\left(\left|\nabla v_{n}\right|^{2}+V_{\lambda_{n}} g^{2}\left(v_{n}\right)\right) d x-\int_{\mathbb{R}^{N}} F\left(g\left(\left(v_{n}\right)_{+}\right)\right) d x \\
& \geq\left(\frac{1}{2}-\frac{1}{\theta}\right) \int_{B_{\ell}\left(x_{n}\right) \cap\left\{x \mid a(x) \geq M_{0}\right\}}\left(\lambda_{n} a(x)+1\right) g^{2}\left(v_{n}\right) d x \\
& \geq\left(\frac{1}{2}-\frac{1}{\theta}\right) \int_{B_{\varrho}\left(x_{n}\right) \cap\left\{x \mid a(x) \geq M_{0}\right\}} \lambda_{n} a(x)\left|g\left(v_{n}\right)-g(v)\right|^{2} d x
\end{aligned}
$$




$$
\begin{aligned}
\geq & \left(\frac{1}{2}-\frac{1}{\theta}\right) \lambda_{n} M_{0} \\
& \times\left(\int_{B_{\varrho}\left(x_{n}\right)}\left|g\left(v_{n}\right)-g(v)\right|^{2} d x-\int_{B_{\varrho}\left(x_{n}\right) \cap\left\{x \mid a(x) \leq M_{0}\right\}}\left|g\left(v_{n}\right)-g(v)\right|^{2} d x\right) \\
= & \left(\frac{1}{2}-\frac{1}{\theta}\right) \lambda_{n} M_{0}\left(\int_{B_{\varrho}\left(x_{n}\right)}\left|g\left(v_{n}\right)-g(v)\right|^{2} d x-o(1)\right) \\
\rightarrow & +\infty \quad(n \rightarrow \infty),
\end{aligned}
$$

which shows that $g\left(v_{n}\right) \rightarrow g(v)$ in $L^{q}\left(\mathbb{R}^{N}\right)$ for $2<q<2$. In the above proof, we have used the fact that $\mu\left\{B_{\varrho}\left(x_{n}\right) \cap\left\{x \mid a(x) \leq M_{0}\right\}\right\} \rightarrow 0$ as $n \rightarrow \infty$ and the $L^{2}$ bounded property of $g\left(v_{n}\right)$.

Now, since $\left\{v_{n}\right\}$ is bounded, by the Fatou lemma, we obtain

$$
\begin{aligned}
& \int_{\Omega}|\nabla v|^{2} d x \leq \lim _{n \rightarrow \infty} \int_{\mathbb{R}^{N}}\left|\nabla v_{n}\right|^{2} d x, \\
& \int_{\Omega} g^{\prime}(v) g(v) v d x \leq \lim _{n \rightarrow \infty} \int_{\mathbb{R}^{N}} g^{\prime}\left(v_{n}\right) g\left(v_{n}\right) v_{n} d x .
\end{aligned}
$$

But, by the choice of $v_{n}$, we have

$$
\int_{\mathbb{R}^{N}}\left(\left|\nabla v_{n}\right|^{2}+\left(\lambda_{n} a+1\right) g\left(v_{n}\right) g^{\prime}\left(v_{n}\right) v_{n}\right) d x=\int_{\mathbb{R}^{N}} f\left(g\left(\left(v_{n}\right)_{+}\right)\right) g^{\prime}\left(\left(v_{n}\right)_{+}\right) v_{n} d x,
$$

hence,

$$
\begin{aligned}
\int_{\Omega}\left(|\nabla v|^{2}+g(v) g^{\prime}(v) v\right) d x & \leq \lim _{n \rightarrow \infty} \int_{\mathbb{R}^{N}}\left|\nabla v_{n}\right|^{2} d x+\lim _{n \rightarrow \infty} \int_{\mathbb{R}^{N}} g\left(v_{n}\right) g^{\prime}\left(v_{n}\right) v_{n} d x \\
& \leq \lim _{n \rightarrow \infty} \int_{\mathbb{R}^{N}} f\left(g\left(\left(v_{n}\right)_{+}\right)\right) g^{\prime}\left(\left(v_{n}\right)_{+}\right) v_{n} d x .
\end{aligned}
$$

In the following, we will prove that

$$
\int_{\mathbb{R}^{N}}\left|f\left(g\left(\left(v_{n}\right)_{+}\right)\right) g^{\prime}\left(\left(v_{n}\right)_{+}\right) v_{n}-f\left(g\left(v_{+}\right)\right) g^{\prime}\left(v_{+}\right) v\right| d x \rightarrow 0
$$

Indeed,

$$
\begin{aligned}
\int_{\mathbb{R}^{N}} & \left(f\left(g\left(\left(v_{n}\right)_{+}\right)\right) g^{\prime}\left(\left(v_{n}\right)_{+}\right) v_{n}-f\left(g\left(v_{+}\right)\right) g^{\prime}\left(v_{+}\right) v\right) d x \\
= & \int_{\mathbb{R}^{N}}\left(f\left(g\left(\left(v_{n}\right)_{+}\right)\right) g^{\prime}\left(\left(v_{n}\right)_{+}\right) v_{n}-f\left(g\left(v_{+}\right)\right) g^{\prime}\left(\left(v_{n}\right)_{+}\right) v_{n}\right) d x \\
& \quad+\int_{\mathbb{R}^{N}}\left(f\left(g\left(v_{+}\right)\right) g^{\prime}\left(\left(v_{n}\right)_{+}\right) v_{n}-f\left(g\left(v_{+}\right)\right) g^{\prime}\left(v_{+}\right) v\right) d x \\
& :=I_{1}+I_{2} .
\end{aligned}
$$

Since $f\left(g\left(v_{+}\right)\right) g^{\prime}\left(\left(v_{n}\right)_{+}\right) v_{n} \rightarrow f\left(g\left(v_{+}\right)\right) g^{\prime}\left(v_{+}\right) v$, one can easily see that $I_{2} \rightarrow 0$ as $n \rightarrow \infty$, and

$$
\begin{aligned}
I_{1} & =\int_{\mathbb{R}^{N}}\left(f\left(g\left(\left(v_{n}\right)_{+}\right)\right) g^{\prime}\left(\left(v_{n}\right)_{+}\right) v_{n}-f\left(g\left(v_{+}\right)\right) g^{\prime}\left(\left(v_{n}\right)_{+}\right) v_{n}\right) d x \\
& =\int_{\mathbb{R}^{N}}\left(f\left(g\left(\left(v_{n}\right)_{+}\right)\right)-f\left(g\left(v_{+}\right)\right)\right) g^{\prime}\left(\left(v_{n}\right)_{+}\right) v_{n} d x
\end{aligned}
$$




$$
\begin{aligned}
& \leq \int_{\mathbb{R}^{N}}\left|f\left(g\left(\left(v_{n}\right)_{+}\right)\right)-f\left(g\left(v_{+}\right)\right)\right| \cdot\left|v_{n}\right| d x \\
& \leq\left\|f\left(g\left(\left(v_{n}\right)_{+}\right)\right)-f\left(g\left(v_{+}\right)\right)\right\|_{q}\left\|v_{n}\right\|_{q^{\prime}} \quad\left(1 / q+1 / q^{\prime}=1\right) \\
& \rightarrow 0 \quad(n \rightarrow \infty)
\end{aligned}
$$

by using $g\left(v_{n}\right) \rightarrow g(v)$ in $L^{q}\left(\mathbb{R}^{N}\right)$ for $2<q<2^{*}$. It follows from (4.4) that

$$
\int_{\Omega}\left(|\nabla v|^{2}+g(v) g^{\prime}(v) v\right) d x \leq \int_{\mathbb{R}^{N}} f\left(g\left(v_{+}\right)\right) g^{\prime}\left(v_{+}\right) v d x
$$

thus, there exists $0<\alpha_{0} \leq 1$ such that $\alpha_{0} v \in N_{\Omega}$ and

$$
\Phi_{\Omega}\left(\alpha_{0} v\right) \leq \Phi_{\Omega}(v),
$$

hence $c(\Omega) \leq \Phi_{\Omega}\left(\alpha_{0} v\right)<\Phi_{\Omega}(v) \leq \lim _{n \rightarrow \infty} \Phi_{\lambda_{n}}=k<c(\Omega)$. A contradiction. Thus we have proved that $\lim _{\lambda \rightarrow \infty} c_{\lambda} \rightarrow c(\Omega)$ as $\lambda \rightarrow+\infty$.

Proof of Theorem 2.1 Suppose that $\left\{v_{n}\right\}$ is a sequence such that $v_{n} \in N_{\lambda}, \Phi_{\lambda_{n}}\left(v_{n}\right)=c_{\lambda_{n}}$, by the proof of Lemma 3.2, we have $\nabla v_{n} \rightarrow \nabla v$ in $L^{2}\left(\mathbb{R}^{N}\right), g\left(v_{n}\right) \rightarrow g(v)$ in $L^{q}\left(\mathbb{R}^{N}\right)$ for $2<q<2^{*}$ and $\left.v\right|_{\Omega^{c}}=0$. Moreover, $\Phi_{\Omega}(v) \leq \lim _{n \rightarrow \infty} \Phi_{\lambda_{n}}\left(v_{n}\right) \leq c(\Omega)$, and if $v \in N_{\Omega}$, then $\Phi_{\Omega}(v)=c(\Omega)$. Hence, in the following, we need only to prove that $v \in N_{\Omega}$. To do this, it is sufficient to prove that

$$
\begin{aligned}
& \lim _{n \rightarrow \infty} \int_{\mathbb{R}^{N}} \lambda_{n} a(x) g^{2}\left(v_{n}\right) d x=0, \\
& \lim _{n \rightarrow \infty} \int_{\mathbb{R}^{N}}\left|\nabla v_{n}\right|^{2} d x=\int_{\mathbb{R}^{N}}|\nabla \nu|^{2} d x
\end{aligned}
$$

and

$$
\lim _{n \rightarrow \infty} \int_{\mathbb{R}^{N}} g\left(v_{n}\right) g^{\prime}\left(v_{n}\right) v_{n} d x=\int_{\mathbb{R}^{N}} g(v) g^{\prime}(v) v d x .
$$

In fact, if one of the above three limits does not hold, by the Fatou lemma, we have

$$
\int_{\mathbb{R}^{N}}|\nabla v|^{2} d x+\int_{\mathbb{R}^{N}} g(v) g^{\prime}(v) v d x<\int_{\mathbb{R}^{N}} f(g(v)) g^{\prime}(v) v d x .
$$

Similar to above, there exists $\alpha_{0} \in(0,1)$ such that $\alpha_{0} v \in N_{\Omega}$ and $c(\Omega) \leq \Phi_{\Omega}\left(\alpha_{0} v\right)<$ $\Phi_{\Omega}(v) \leq \lim _{n \rightarrow \infty} \Phi_{\lambda_{n}}\left(v_{n}\right) \leq c(\Omega)$. A contradiction, and thus we complete the proof of Theorem 2.1.

\section{Competing interests}

The author declares that she has no competing interests.

\section{Acknowledgements}

The author would like to thank the referee for some valuable comments and helpful suggestions. This study was supported by the National Natural Science Foundation of China (11161041, 31260098) and the Fundamental Research Funds for the Central Universities (zyz2012080, zyz2012074). 


\section{References}

1. Bartsch, T, Wang, Z: Multiple positive solutions for a nonlinear Schrödinger equation. Z. Angew. Math. Phys. 51 , 366-384 (2000)

2. Kurihura, S: Large-amplitude quasi-solitons in superfluid. J. Phys. Soc. Jpn. 50, 3262-3267 (1981)

3. Laedke, EW, Spatschek, KH, Stenflo, L: Evolution theorem for a class of perturbed envelop soliton solutions. J. Math. Phys. 24, 2764-2769 (1983)

4. Brandi, HS, Manus, C, Mainfray, G, Lehner, T, Bonnaud, G: Relativistic and ponderomotive self-focusing of a laser beam in a radially inhomogeneous plasma. Phys. Fluids B 5, 3539-3550 (1993)

5. Chen, $\mathrm{XL}$, Sudan, RN: Necessary and sufficient conditions for self-focusing of short ultraintense laser pulse. Phys. Rev. Lett. 70, 2082-2085 (1993)

6. De Bouard, A, Hayashi, N, Saut, JC: Global existence of small solutions to a relativistic nonlinear Schrödinger equation Commun. Math. Phys. 189, 73-105 (1997)

7. Ritchie, B: Relativistic self-focusing channel formation in laser-plasma interactions. Phys. Rev. E 50, $687-689$ (1994)

8. Poppenberg, M, Schmitt, K, Wang, Z: On the existence of solutions to quasilinear Schrödinger equation. Calc. Var. Partial Differ. Equ. 14, 329-344 (2002)

9. Liu, J, Wang, Y, Wang, Z: Soliton solutions for quasilinear Schrödinger equation II. J. Differ. Equ. 187, 473-493 (2003)

10. Colin, M, Jeanjean, L: Solutions for a quasilinear Schrödinger equation: a dual approach. Nonlinear Anal. 56, 213-226 (2004)

11. Berestycki, H, Lions, PL: Nonlinear scalar field equations I. Arch. Ration. Mech. Anal. 82, 313-346 (1983)

12. Lions, PL: The concentration-compactness principle in the calculus of variations. The locally compact case. Part I. Ann. Inst. Henri Poincaré, Anal. Non Linéaire 1, 109-145 (1984)

13. Guo, Y, Tang, Z: Ground state solutions for the quasilinear Schrödinger equation. Nonlinear Anal. 75, 3235-3248 (2012)

14. Guo, Y, Tang, Z: Ground state solutions for the quasilinear Schrödinger systems. J. Math. Anal. Appl. 389, 322-339 $(2012)$

15. Colin, M, Jeanjean, L, Squassina, M: Stability and instability results for standing waves of quasi-linear Schrödinger equations. Nonlinearity 23, 1353-1385 (2010)

16. Ambrosetti, A, Badiale, M, Cingolani, S: Semiclassical states of nonlinear Schrödinger equations. Arch. Ration. Mech. Anal. 140, 285-300 (1997)

17. Ambrosetti, A, Malchiodi, A, Secchi, S: Multiplicity results for some nonlinear Schrödinger equations with potentials. Arch. Ration. Mech. Anal. 159, 253-271 (2001)

18. Byeon, J, Wang, Z: Standing waves with a critical frequency for nonlinear Schrödinger equations II. Calc. Var. Partial Differ. Equ. 18, 207-219 (2003)

19. Cingolani, S, Lazzo, M: Multiple positive solutions to nonlinear Schrödinger equations with competing potential functions. J. Differ. Equ. 160, 118-138 (2000)

20. Cingolani, S, Nolasco, M: Multi-peaks periodic semiclassical states for a class of nonlinear Schrödinger equations. Proc R. Soc. Edinb. 128, 1249-1260 (1998)

21. Del Pino, M, Felmer, P: Semi-classical states for nonlinear Schrödinger equations. Ann. Inst. Henri Poincaré 15, 127-149 (1998)

22. Del Pino, M, Felmer, P: Multi-peak bound states for nonlinear Schrödinger equations. J. Funct. Anal. 149, 245-265 (1997)

23. Floer, A, Weinstein, A: Nonspreading wave packets for the cubic Schrödinger equation with a bounded potential. J. Funct. Anal. 69, 397-408 (1986)

24. Oh, YG: On positive multi-bump bound states of nonlinear Schrödinger equations under multiple well potential. Commun. Math. Phys. 131, 223-253 (1990)

25. Oh, YG: Existence of semiclassical bound states of nonlinear Schrödinger equations with potentials of class $(V)_{a}$. Commun. Partial Differ. Equ. 13, 1499-1519 (1988)

26. Rao, M, Ren, Z: Theory of Orlicz Space. Dekker, New York (1991)

doi:10.1186/1687-2770-2013-9

Cite this article as: Jiao: Least energy solutions for a quasilinear Schrödinger equation with potential well. Boundary Value Problems 2013 2013:9.

\section{Submit your manuscript to a SpringerOpen ${ }^{\ominus}$ journal and benefit from:}

- Convenient online submission

- Rigorous peer review

- Immediate publication on acceptance

- Open access: articles freely available online

- High visibility within the field

- Retaining the copyright to your article

Submit your next manuscript at $>$ springeropen.com 